\title{
Brachial Plexus Ultrasound and MRI in Children with Brachial Plexus Birth Injury
}

\author{
(D) A. Gunes, (D)E. Bulut, (D)A. Uzumcugil, and (D) K.K. Oguz
}

\begin{abstract}
BACKGROUND AND PURPOSE: Brachial plexus birth injury is caused by traction on the neck during delivery and results in flaccid palsy of an upper extremity commonly involving C5-C6 nerve roots. MR imaging and MR myelography help to assess the anatomic location, extent, and severity of brachial plexus injuries which influence the long-term prognosis along with the surgical decision making. Recently, sonography has been increasingly used as the imaging modality of choice for brachial plexus injuries. The aim of this study was to assess the degree of correlation among brachial plexus sonography, MR imaging, and surgical findings in children with brachial plexus birth injury.
\end{abstract}

MATERIALS AND METHODS: This prospective study included 55 consecutive patients (girls/boys = 32:23; mean age, $2.1 \pm 0.8$ months) with brachial plexus birth injury between May 2014 and April 2017. The patients were classified according to the Narakas classification and were followed up at 4- to 6-week intervals for recovery by the Modified Mallet system and sonography without specific preparation for evaluation. All patients had MR imaging under general anesthesia. Nerve root avulsion-retraction, pseudomeningocele, and periscalene soft tissue were accepted brachial plexus injury findings on imaging. Interobserver agreement for MR imaging and the agreement between imaging and surgical findings were estimated using the $\kappa$ statistic. The diagnostic accuracy of sonography and MR imaging was calculated on the basis of the standard reference, which was the surgical findings.

RESULTS: Forty-three patients had pre- and postganglionic injury, 12 had only postganglionic injury findings, and $47 \%$ of patients underwent an operation. On sonography, no patients had preganglionic injury, but all patients had postganglionic injury findings. For postganglionic injury, the concordance rates between imaging and the surgical findings ranged from $84 \%$ to $100 \%$, and the diagnostic accuracy of sonography and MR imaging was $89 \%$ and $100 \%$, respectively. For preganglionic injury, the diagnostic accuracy of MR imaging was $92 \%$. Interobserver agreement and the agreement between imaging and the surgical findings were almost perfect for postganglionic injury $(\kappa=$ $0.81-1, P<.001)$.

CONCLUSIONS: High-resolution sonography can identify and locate the postganglionic injury associated with the upper and middle trunks. The ability of sonography to evaluate pre- and the postganglionic injury associated with the lower trunk was quite limited. Sonography can be used as a complement to MR imaging; thus, the duration of the MR imaging examination and the need for sedation can be reduced by sonography.

ABBREVIATIONS: $\mathrm{BP}=$ brachial plexus; $\mathrm{GI}=$ ganglionic injury; $\mathrm{US}=$ ultrasound; $\mathrm{PST}=$ periscalene soft tissue

B rachial plexus (BP) birth injury is caused by traction on the neck during delivery and results in flaccid palsy of an upper extremity commonly involving the C5-C6 nerve roots. ${ }^{1}$ Imaging

Received March 9, 2018; accepted after revision June 25.

From the Department of Radiology (A.G.), University of Health Sciences, Ankara Child Health and Diseases Hematology Oncology Training and Research Hospital, Ankara, Turkey; and Departments of Radiology (E.B., K.K.O.) and Orthopedics and Traumatology (A.U.), Hacettepe University Faculty of Medicine, Ankara, Turkey.

Please address correspondence to Altan Gunes, MD, Department of Radiology, University of Health Sciences, Ankara Child Health and Diseases Hematology Oncology Training and Research Hospital, Dıskapı, 06130, Ankara, Turkey; e-mail: draltangunes@gmail.com

$\equiv$ Indicates article with supplemental on-line tables. studies help to assess the anatomic location, extent, and severity of $\mathrm{BP}$ injuries, which influence the long-term prognosis along with the surgical decision-making. ${ }^{1} \mathrm{MR}$ imaging has been the preferred imaging technique for the evaluation of BP injuries with a high diagnostic accuracy (87\%) and the ability to differentiate pre- and postganglionic injuries (GIs). ${ }^{2-4}$ MR myelography is even superior to conventional MR imaging in detecting root avulsions, with a diagnostic accuracy of $92 \% .^{5,6}$ Because of the ease of availability and superior spatial resolution for a quick, real-time evaluation of nerves without sedation or contrast administration,

http://dx.doi.org/10.3174/ajnr.A5749 
ultrasound (US) has been increasingly used as the imaging technique of choice in recent years. ${ }^{7-9}$ US can detect nerve injury in the form of a neuroma and/or scar tissue formation. ${ }^{9,10}$ Detection of nerve root injuries has been reported to be $100 \%$ for C5-C7, 84\% for C8, and $64 \%$ for $\mathrm{T} 1$ in adult patients. ${ }^{11}$ These nerve roots can also be evaluated in children with similar detection rates except the $\mathrm{C} 8$ and T1 nerve roots because of difficulty in evaluating C 8 and T1. ${ }^{9}$

The aim of this study was to assess the degree of correlation among BP US, MR imaging, and surgical findings in children with BP birth injury.

\section{MATERIALS AND METHODS Patients}

The study was approved by the local ethics committee (Hacettepe University Faculty of Medicine Ethics Committee, Reference Number $=16969557-627$ ), and the families of patients gave written informed consent. This prospective study included 55 patients (girls/boys $=32: 23$; mean age, $2.1 \pm 0.8$ months; range, $0.5-3$ months) who were referred with the clinical diagnosis of BP birth injury from the Department of Orthopedics and Traumatology, between May 1, 2014, and April 1, 2017. The diagnosis was based on the clinical examination and risk factors (maternal diabetes $[n=5]$, high birth weight $[n=21]$, prolonged labor $[n=8]$, and assisted or difficult deliveries $[n=5])$. Inclusion criteria for the patients in the study were having a BP birth injury and having a BP US and MR imaging studies with diagnostic quality. Exclusion criteria for the patients in the study included having inadequate medical records, not having regular follow-up, and having suboptimal/inadequate US and MR imaging scans due to motion or breathing artifacts.

\section{Clinical Follow-Up of Patients}

The patients were classified according to the Narakas classification $^{12}$ of obstetric BP palsy: grade $\mathrm{I}=20$, grade $\mathrm{II}=21$, grade $\mathrm{III}=2$, and grade IV $=12$. The patients were followed up to 2 years of age at 4- to 6-week intervals for recovery by the Modified Mallet system and US. ${ }^{1}$ The same orthopedist (A.U.), with 11 years of experience in managing BP birth injury, examined and followed the patients throughout the study. Our clinical approach was consistent with that introduced by Gilbert et al. ${ }^{13}$ The patients who demonstrated recovery in biceps function within the first 12 weeks of life were followed conservatively with functional rehabilitation. If the biceps function of the patients did not demonstrate recovery at 12 weeks, these patients were considered as possible surgical candidates, and the definitive surgery decision for the patients was made at 6 months. If the patient had total paralysis, especially when associated with Horner syndrome and no recovery at 3 months or insufficient recovery at 6 months, the operation would almost certainly to be required. The surgical intervention for all patients was performed by the same orthopedist (A.U.). No surgical laminectomies were performed to determine the nerve root avulsion to avoid increased morbidity and mortality of this procedure. ${ }^{14}$ The nerve root integrity was determined by intraoperative neurophysiologic studies in which stimulating electrodes were used to observe the neuroelectrical responses. In these studies, the inability to receive a motor response distal to the nerve roots was considered a nerve root injury.

\section{Sonographic Technique}

The US examinations were performed by 1 pediatric radiologist (A.G.), who had 7 years of experience in musculoskeletal imaging and US. The radiologist was not blinded to the indication for US but was blinded to the findings of the physical examination. All patients were examined with a high-frequency linear probe (Sonoline G40, Siemens, Erlangen, Germany [5-7.5 MHz]; Xario, Toshiba, Tokyo, Japan [5-12 MHz]) in a supine and contralateral (unaffected side) decubitus position without specific preparation for US. The examinations took an average of 5 minutes for each patient. The protocol of BP US was standardized and based on the detection of the anatomic landmarks in the neck such as the vertebral artery (for nerve root) and scalene muscles (for nerve root and trunk). ${ }^{8,15}$ The probe was placed above the clavicle, and it was advanced from the supraclavicular area to the sternocleidomastoid muscle for evaluation of the interscalene-supraclavicular regions and the neural foraminal region in transverse and longitudinal views. The predefined findings of the nerve root avulsion, which include nonvisualization of the nerves on the affected side, pseudomeningocele (CSF collections due to dural tears in proximity to intervertebral foramina), and periscalene soft tissue (PST) that represent a posttraumatic neuroma or scar tissue, were evaluated on the affected side by US. ${ }^{2,6,16}$ PST is defined as the asymmetric linear and/or nodular thickening of the nerve root trunks detected in the interscalene space. ${ }^{16}$ The nerve root avulsion and pseudomeningocele were the accepted findings of preGI, and the PST was accepted as a sign of a post-GI finding. ${ }^{2,6,16}$ The location, extension, size, echogenicity, and vascularity of the PSTs were evaluated. The size of the PSTs was measured in the transverse section. The changes in size and echogenicity of PSTs were followed up by the same radiologist (A.G.) throughout the study.

\section{MR Imaging Technique}

All MR imaging examinations were performed on a 1.5T scanner (Symphony; Siemens) with a head and neck coil. Our MR imaging protocol for BP applied the conventional (spin-echo T1WI, TSE T2WI, STIR) and CISS sequences. No paramagnetic contrast agent was used. Both the left and right BPs were imaged to allow comparison and better detection of the abnormalities. The imaging technique included axial (C3 to inferior axilla) and coronal oblique (including both shoulders) T1WI (TR/TE $=450-600$ / $12-15 \mathrm{~ms}$, FOV $=16 \mathrm{~cm}$, section thickness $/$ gap $=3 / 1 \mathrm{~mm}$ ); axial and sagittal T2WI for the cervical spine; coronal oblique fat-suppressed T2WI for the BP (TR/TE/TI $=3600-4000 / 70-80 / 160$ $\mathrm{ms}$, FOV $=16-20 \mathrm{~cm}$, section thickness $/$ gap $=3 / 1 \mathrm{~mm}$ ); and axial and coronal CISS $(\mathrm{TR} / \mathrm{TE}=7.6 / 3.2 \mathrm{~ms}, \mathrm{FOV}=16-18 \mathrm{~cm}$, section thickness/gap $=0.7-1 / 0.5 \mathrm{~mm})$. The examinations took an average of 25-30 minutes, including the preparation of the patient. Each patient was scanned under general anesthesia. MR imaging of pre-GI can show the partial (ventral or dorsal rootlets) or complete nerve root avulsion seen as the discontinuity of the roots, nerve root retraction, displacement and/or signal abnormalities of cord, and pseudomeningocele. ${ }^{2,6} \mathrm{MR}$ imaging of post-GI shows the asymmetric linear and/or nodular thickening of the nerve roots and trunks that is detected in the interscalene space with imaging defined as a PST. ${ }^{16}$ The location, extension, size, and the intensity of the PSTs were evaluated. The size of the PST was measured in the axial image. 

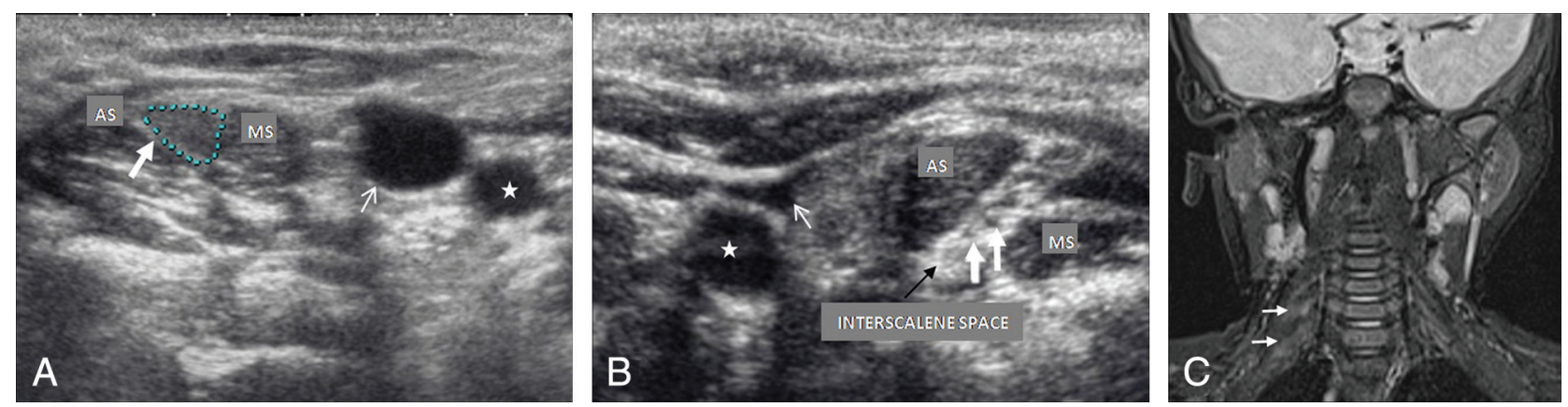

FIG 1. Case 25, in a 1-month-old infant with right-sided total brachial plexus paralysis with homogeneous periscalene soft tissue at the C5-7 level (arrow) between the anterior (AS) and middle scalene (MS) muscles on a transverse sonographic image (A). The thin arrow and star show the right internal jugular vein and carotid artery, respectively, on image $A$. $B$, The transverse scan sonographic image shows the normal interscalene space (black arrow) and nerve roots as hypoechoic oval cross-sections (white arrows) between the AS and MS muscles. The thin arrow and star show left internal jugular vein and carotid artery, respectively, on B. C, Coronal oblique fat-suppressed TSE T2WI shows periscalene soft tissues (arrows) coursing through the right interscalene space at the C5-7 level, findings similar to those in $A$.
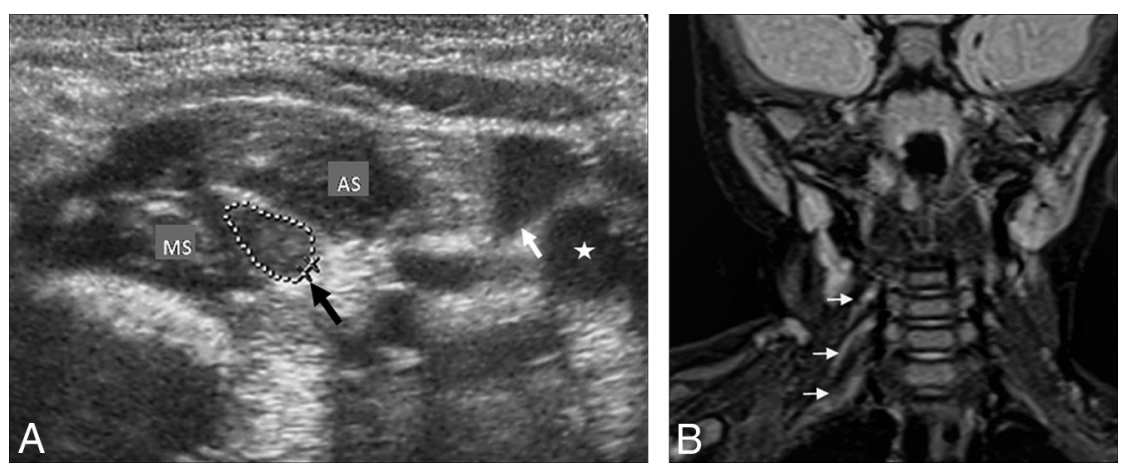

FIG 2. Case 20, a 2-month-old female patient with a right brachial plexus birth injury. A, Transverse scan sonography of the interscalene space shows homogeneous echogenicity periscalene soft tissue with fusiform morphology (black arrow) between the anterior (AS) and middle scalene (MS) muscles at the C4-7 level. The white arrow and star show the right internal jugular vein and carotid artery, respectively. B, Coronal oblique fat-suppressed TSE T2WI shows periscalene soft tissues (arrows) coursing through the right interscalene space at the $C 4-7$ level, findings similar to those in $A$.

\section{Analysis of Findings}

All patients had MR imaging (mean time $=5.4$ months [range, 2-11 months]) after the US examinations (except 4 patients) because the BP birth injury is a medicolegal problem in our country and MR imaging can demonstrate this injury. All MR imaging was analyzed independently, without knowledge of the side of the injury, clinical and US findings, by 2 radiologists (E.B. with 8 years of experience in neuroimaging, and K.K.O. with 17 years of experience in neuroimaging). Finally, the degree of correlation among the clinical, imaging, and surgical findings was analyzed by all investigators in consensus. The surgical and histopathologic findings were the standard of reference for patients who underwent an operation. The standard reference was clinical follow-up for the patients who did not undergo surgery. No patient was excluded from the study for suboptimal US and MR imaging evaluation.

\section{Statistical Analysis}

Comparative analysis was performed using a $\chi^{2}$ test for categoric variables and Mann-Whitney $U$ and Kruskal-Wallis tests for nonnormally distributed continuous variables. Interobserver agreement for MR imaging and the agreement between imaging and surgical findings were estimated using the $\kappa$ statistic (range, -1 to +1 ), which is interpreted as follows: $<0.40$, poor to fair agree- ment; 0.41-0.60, moderate agreement; 0.61-0.80, substantial agreement; and 0.81-1.00, almost perfect agreement. The sensitivity, specificity, positive and negative predictive values, and accuracy were calculated for both US and MR imaging for the detection of pre- and post-GI using a $2 \times 2$ table based on the surgical findings. The statistical analysis was conducted with statistical software (SPSS, Version 21.0; IBM, Armonk, New York). A $P$ value $<.05$ was considered statistically significant.

\section{RESULTS}

Forty-three patients had pre- and postGI, and 12 had only post-GI. Twenty-six of 55 patients (47\%) underwent an operation ( 24 with pre- and post-GI and 2 with only post-GI). There was no significant difference in the mean age between girls ( $2.1 \pm 0.7$ months; range, $0.5-3$ months) and boys ( $2 \pm 0.9$ months; range, $0.5-3$ months $)(P=.94)$. No significant difference was found in the mean birth weight between girls (3865 g; interquartile range, $3600-4035 \mathrm{~g}$ ) and boys (4000 g; interquartile range, $3830-4125 \mathrm{~g})(P=.05)$. According to the Modified Mallet scoring system, the mean global abduction $\left(72.1^{\circ}\right.$ [range, $0^{\circ}-130^{\circ}$ ] $)$ and external rotation $\left(22.6^{\circ}\right.$ [range, $0^{\circ}-90^{\circ}$ ] scores were 2.57 and 2.22, respectively. The mean Modified Mallet scores for the ability to bring the hand to the neck, to the back, and to the mouth were $2.12,2.46$, and 2.50 , respectively. Twelve patients had the Horner sign. Baseline characteristics, imaging findings, and the follow-up data of patients are presented in the Online Table.

All patients had PST on the affected side with no detectable pre-GI on US (Figs 1 and 2). The PST appeared as a smooth well-defined solid mass that usually extended laterally to the BP trunk region on US, with no internal vascularity. The echogenicity of PST $(90 \%, 114 / 127)$ was usually similar to that of the scalene muscles, but some lesions had mixed echotexture (10\%, 13/127). US revealed 127 PSTs that showed the affected number of nerve roots in 55 patients. The mean caliber of the PST was $6.6 \pm 1.9$ 
$\mathrm{mm}$ (range, 3.4-10.2 $\mathrm{mm}$ ), and the thickness of the PST was not significantly different among the Narakas groups $(P=.26)$ and between US and MR imaging examination $(6.1 \pm 2 \mathrm{~mm}$ [range, 3-11.6 mm]), $(P=.34)$. Although the thickness of all PSTs was reduced in the follow-up period (at first evaluation, $6.3 \pm 1.8 \mathrm{~mm}$, [range, 3.3-9.6 mm], at the second evaluation, $6.1 \pm 1.7 \mathrm{~mm}$ [range 3.1-9.3 mm]), PSTs remained persistent thereafter. All PSTs also did not show cystic/hemorrhagic degeneration or calcification on follow-up.

All patients had abnormal MR imaging findings on the affected side. The nerve root avulsion/retraction was seen in 43 patients in at least 1 level $(n=25)$ on MR imaging, and root avulsion without associated pseudomeningocele was seen in 4 patients (Fig 3A). The surgical electrophysiologic examination did not confirm the root avulsion diagnosis in 2 patients with a positive MR imaging finding, case 5 (C5) and case 49 (C5 and C6), and it revealed avulsion in 3 patients with negative MR imaging findings, cases 37 and 45 (C7, C8, and T1) and case 52 (C5). In clinical terms, there were also discrepancies between clinical findings and MR imaging in these patients (cases 5, 37, 45, 49, and 52). The pseudomeningocele without associated root avulsion was seen in 3 patients (cases 33, 39, and 42), and nerve root avulsion in the pseudomeningocele was not evaluated optimally in 3 patients (cases 5, 37, and 45) (Fig 3B). MR imaging showed 139 PSTs that usually showed signal similar to that of scalene muscles (93\%, $129 / 139)$ on T1WI and higher signal $(100 \%, 139 / 139)$ than muscles on T2WI. The locations and extensions of the PST were similar to those of US findings. The US and MR imaging showed concordance in $85 \%(47 / 55)$ of patients with PST $(91 \%, 127 / 139)$ except in 8 cases (C8 $[n=4]$ and T1 $[n=8]$ ) (Figs $1 C$ and $2 B$ ). The clinical, US, and MR imaging findings were concordant in $85 \%$ of patients $(47 / 55)$ with post-GI.

In the surgically proved cases, for the post-GI, the sensitivity, specificity, positive and negative predictive values, and diagnostic
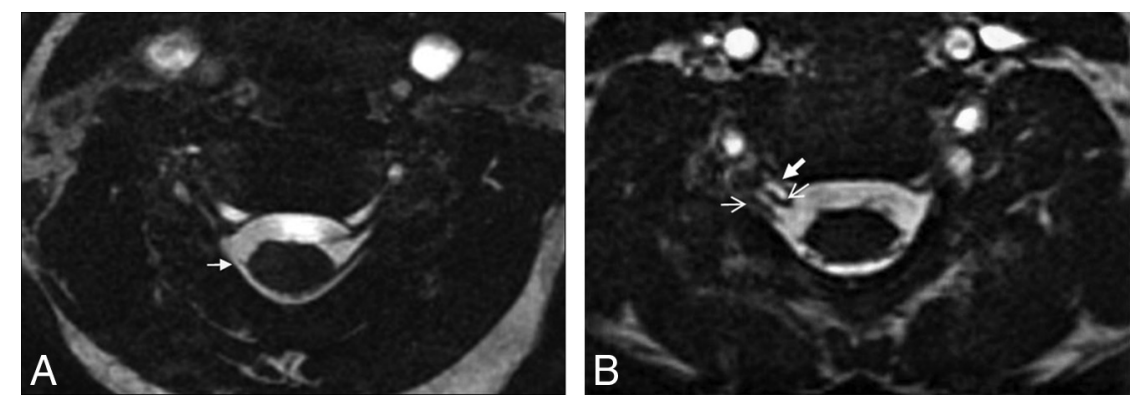

FIG 3. Case 31, in a 0.5 -month-old male patient with right brachial plexus birth injury. C7 root avulsions (arrow) without associated pseudomeningocele were seen in the axial 3D-CISS image (A). Case 42, a 2-month-old female patient with right brachial plexus birth injury. The pseudomeningocele (thick arrow) without associated root avulsion (thin arrows) was seen at the C6-7 level in the axial 3D-CISS image (B).

Sensitivity, specificity, positive and negative predictive values, and diagnostic accuracy of US and MRI in the surgically proven cases

\begin{tabular}{lcccrr}
\hline & Sensitivity \% & Specificity \% & PPV \% & NPV \% & Accuracy \% \\
\hline $\begin{array}{l}\text { For post-ganglionic injury } \\
\text { US }\end{array}$ & $84(66 / 78)$ & $100(52 / 52)$ & $100(66 / 66)$ & $81(52 / 64)$ & $90(118 / 130)$ \\
$\quad$ MRI & $100(78 / 78)$ & $100(52 / 52)$ & $100(78 / 78)$ & $100(52 / 52)$ & $100(130 / 130)$ \\
$\begin{array}{l}\text { For pre-ganglionic injury } \\
\quad \text { MRI }\end{array}$ & $84(37 / 44)$ & $96(83 / 86)$ & $92(37 / 40)$ & $92(83 / 90)$ & $92(120 / 130)$ \\
\hline
\end{tabular}

Note:-PPV indicates positive predictive value; NPV, negative predictive value. accuracy of US ranged from $81 \%$ to $100 \%$ (Table). The sensitivity, specificity, positive and negative predictive values, and the diagnostic accuracy of MR imaging ranged from $84 \%$ to $100 \%$ for preand post-GI (Table). The agreement among the US, MR imaging, and surgical findings was almost perfect for post-GI $(\kappa=0.81$ and 1 , respectively; $P<.001)$. For pre- and post-GI, the $\kappa$ values between observers were 0.89 and $0.93(P<.001)$, and there was almost perfect agreement.

Twenty-six surgical procedures were performed during the 3 -year study period (mean time $=6.6 \pm 1.1$ months, [range, 5-9 months]; neurolysis [ $n=19]$, nerve grafting [ $n=5]$, and neurotization $[n=2]$ ) without any surgical or early postoperative complications. Histopathologic studies were available in 9 patients: Five lesions were compatible with scar tissue, and 4 lesions were compatible with posttraumatic neuroma. The early results of shoulder and elbow function recovery demonstrated 66\% success at 9 months postoperatively. In 29 patients who did not undergo an operation, the biceps function was recovered before 6 months in $22(75 \%)$ patients and after 6 months in 7 patients (24\%). Patients with C5-C6 palsy $(90 \%, 18 / 20)$ had a statistically higher spontaneous functional recovery rate than patients with C5-C7 palsy (52\%, $11 / 21)(P=.008)$. No patients with C5-T1 palsy had spontaneous

\section{DISCUSSION}

The etiology and mechanism of BP birth injury is not completely known, though many maternal and fetal factors have been suggested as the cause such as shoulder dystocia and high birth weight. ${ }^{17,18}$ Shoulder dystocia has been identified as the greatest risk factor in the etiology of BP birth injury in our study. Birth weight higher than $4500 \mathrm{~g}$ is the most important fetal factor for BP birth injury, increasing the risk by 10 -fold. ${ }^{18}$ Yet, in our study, the mean birth weight (3863 g) was $<4500 \mathrm{~g}$, and some BP birth injuries occurred in women without identifiable risk factors. In agreement with previous data, these findings suggest the unpredictability of BP birth injury occurrence. $^{17,18}$

In our study, most of the infants (74\%) had paralysis of the C5-C6 \pm C7 roots, similar to findings in the previous report by Kozin. ${ }^{19}$ Our study included patients with a diagnosis of BP birth injury on clinical grounds. Therefore, as expected, all patients had abnormal findings on the affected side on US. In these patients, US effectively showed the post-GI at the interscalene space, in concordance with MR imaging (82\%-91\%) and the surgical findings (90\%). How- 
ever, although all post-GIs at the C4-C7 levels were detected, US failed at the $\mathrm{T} 1$ and occasionally the $\mathrm{C} 8$ nerve root due to an improper window for the examination in 8 patients. In addition, the short necks of the patients and nerve root-bone relationship made evaluation difficult. Consequently, the lower nerve roots and trunks were not evaluated optimally in about $60 \%$ of patients. Previous studies with US mentioned similar technical challenges. $^{7-9}$

The posttraumatic neuroma is a disorganized proliferation of regenerating axons at the proximal stump of a transected nerve, corresponding to lesion type III or IV in the Sunderland classification. ${ }^{20}$ Thickening of the nerve root trunks might be related to neuroma and/or scar tissue, and the differentiation between them can be difficult by imaging methods. In the literature, the US and MR imaging criteria for the differential diagnosis between traction injury and neuroma have not been specified. We also could not find a distinguishing feature on US and MR imaging, in terms of the echogenicity, intensity, and size of the lesions, either at the initial diagnosis or during the follow-ups. Similarly, differentiation of the scar tissue from a neuroma was not possible in the studies by Abbott et $\mathrm{al}^{3}$ and Wandler et al. ${ }^{16}$ Therefore; imaging does not obviate the role of histopathologic examination for definitive diagnosis of these lesions.

Contrary to the post-GI, for the pre-GI, the US findings were not concordant with MR imaging and the surgical findings. This finding may be due to the technical insufficiency of US related to the artifacts caused by the transverse process of vertebrae. It has been reported that US can reveal root avulsion after traumatic BP injuries in adults. ${ }^{21}$ However, the artifacts caused by vertebrae make it difficult to see intraspinal-intraforaminal injuries despite the high-frequency probes. Thus, US has a very limited value for showing the pre-GI and full extent of the injury. However, MR myelography is superior in the assessment of pre-GI compared with US because it allows obtaining high-quality, detailed anatomic images of the intraspinal-intraforaminal contents. Although MR myelography may have limitations related to CSF flow artifacts in showing the root avulsion, it is an effective method for demonstrating not only root avulsion but also the level of the injury. ${ }^{6}$ Our study showed that MR myelography was successful in depicting the presence of the surgically proved root avulsion, and this finding was similar to those in previous studies. $^{6,22}$ The diagnostic accuracy of MR imaging was $92 \%$ in our study, which is also compatible with that in previous reports. ${ }^{4} \mathrm{~A}$ pseudomeningocele can be seen with or without root avulsion, and the presence of a pseudomeningocele is highly indicative but not pathognomonic for a pre-GI. ${ }^{3,23}$ There were 3 cases with pseudomeningocele but without root avulsion (cases 33, 39, and 42 ) in the present cohort.

In our study, those patients (75\%) who recovered the upper trunk muscle strength spontaneously in the first 6 months of life had a complete neurologic recovery during the first 2 years of life, which is compatible with previous study findings. ${ }^{12,24}$ The axon and its myelin covering (endoneurium) lose continuity with the cell body in grade III injury according to the Sunderland classification. ${ }^{20}$ In addition to grade III injury, the perineurium is disrupted in grade IV injury; however, the nerve is still in continuity and surgical intervention is usually required to re-establish nerve transduction by removing the scar tissue. ${ }^{20}$ In our study, 29 patients who improved spontaneously most likely had grade III injury. Compared with the range of $30 \%-90 \%$ recovery rate in the literature, our finding of 55\% is still below the high expectations quoted in previous studies. ${ }^{25,26}$ The varying degrees of the recovery rates show that patients need to be strictly monitored. For the diagnosis of BP injuries and treatment planning, a clinical assessment needs to be made in conjunction with an imaging examination. At this stage, US can play a complementary role to the clinical findings with its ability to visualize the interscalene space with high resolution. It yields information to clinicians about the presence, localization, and extension of the PST. When there is insufficient or no recovery in patients' follow-ups, MR myelography can be performed with superficial sedation instead of general anesthesia to show the pre-GI with fewer sequences such as STIR and 3D heavily T2WI, which, in turn, shortens the scan time. MR imaging can be performed earlier for patients with total BP palsy.

The strengths of our study are the prospective design, close follow-up of patients with the clinical examination and US, the presence of MR imaging of all patients, and the presence of the surgical findings for comparison. However, there are also some limitations: 1) The enhancement of the intradural nerve root and paraspinal muscles suggest functional impairment of the nerve despite morphologic continuity. ${ }^{20}$ In our study, we did not use paramagnetic contrast agents in any of the examinations; thus, we could not demonstrate the possible damage to the nerve root, which could exist despite morphologic continuity, a form of pre-GI; 2) we did not evaluate the histopathology in all operated patients because of the limited biopsy specimen; 3) we did not evaluate the reproducibility and reliability of the US technique because of our patients' very young age (around 2 months); and 4) the follow-up period of 8 patients was $<1$ year, and these patients are still in follow-up at the time of this writing.

\section{CONCLUSIONS}

High-resolution US can identify and locate the post-GI associated with the upper and middle trunks. The ability of US to evaluate pre- and the post-GI associated with the lower trunk was quite limited. US can be used as a complement to MR imaging; thus, the duration of MR imaging examination and the need for sedation can be reduced by US.

\section{REFERENCES}

1. Abzug JM, Kozin SH. Evaluation and management of brachial plexus birth palsy. Orthop Clin North Am 2014;45:225-32 CrossRef Medline

2. Yoshikawa T, Hayashi N, Yamamoto S, et al. Brachial plexus injury: clinical manifestations, conventional imaging findings, and the latest imaging techniques. Radiographics 2006;26(Suppl 1):S133-43 CrossRef Medline

3. Abbott R, Abbott M, Alzate J, et al. Magnetic resonance imaging of obstetrical brachial plexus injuries. Childs Nerv Syst 2004;20:720-25 CrossRef Medline

4. Tagliafico A, Succio G, Serafini G, et al. Diagnostic accuracy of MRI in adults with suspect brachial plexus lesions: a multicentre retrospective study with surgical findings and clinical follow-up as reference standard. Eur J Radiol 2012;81:2666-72 CrossRef Medline

5. Carvalho GA, Nikkhah G, Matthies C, et al. Diagnosis of root avulsions in traumatic brachial plexus injuries: value of computerized 
tomography myelography and magnetic resonance imaging. J Neurosurg 1997;86:69-76 CrossRef Medline

6. Gasparotti R, Ferraresi S, Pinelli L, et al. Three-dimensional MR myelography of traumatic injuries of the brachial plexus. AJNR Am J Neuroradiol 1997;18:1733-42 Medline

7. Demondion X, Herbinet P, Boutry N, et al. Sonographic mapping of the normal brachial plexus. AJNR Am J Neuroradiol 2003;24: 1303-09 Medline

8. Graif M, Martinoli C, Rochkind S, et al. Sonographic evaluation of brachial plexus pathology. Eur Radiol 2004;14:193-200 CrossRef Medline

9. Smith EC, Xixis KI, Grant GA, et al. Assessment of obstetric brachial plexus injury with preoperative ultrasound. Muscle Nerve 2016;53: 946-50 CrossRef Medline

10. Haber HP, Sinis N, Haerle M, et al. Sonography of brachial plexus traction injuries. AJR Am J Roentgenol 2006;186:1787-91 CrossRef Medline

11. Chen DZ, Cong R, Zheng MJ, et al. Differential diagnosis between preand postganglionic adult traumatic brachial plexus lesions by ultrasonography. Ultrasound Med Biol 2011;37:1196-203 CrossRef Medline

12. Narakas AO. Obstetric brachial plexus injuries. In: Lamb DW, ed. The Paralysed Hand. Edinburgh: Churchill Livingstone; 1987:116-35

13. Gilbert A, Razaboni R, Amar-Khodja S. Indications and results of brachial plexus surgery in obstetrical palsy. Orthop Clin North Am 1988;19:91-105 Medline

14. Amrami K, Port J. Imaging the brachial plexus. Hand Clin 2005;21: 25-37 CrossRef Medline

15. Martinoli C, Bianchi S, Santacroce E, et al. Brachial plexus sonography: a technique for assessing the root level. AJR Am J Roentgenol 2002;179:699-702 CrossRef Medline

16. Wandler E, Lefton D, Babb J, et al. Periscalene soft tissue: the new imaging hallmark in Erb palsy. AJNR Am J Neuroradiol 2010;31: 882-85 CrossRef Medline

17. Foad SL, Mehlman CT, Ying J. The epidemiology of neonatal brachial plexus palsy in the United States. J Bone Joint Surg Am 2008;90: 1258-64 CrossRef Medline

18. Wolf H, Hoeksma AF, Oei SL, et al. Obstetric brachial plexus injury: risk factors related to recovery. Eur J Obstet Gynecol Reprod Biol 2000;88:133-38 CrossRef Medline

19. Kozin SH. Brachial plexus microsurgical indications. J Pediatr Orthop 2010;30:49-52 CrossRef

20. Laurent JP, Lee RT. Birth-related upper brachial plexus injuries in infants: operative and nonoperative approaches. J Child Neurol 1994;9:111-17 CrossRef Medline

21. Gruber H, Glodny B, Galiano K, et al. High-resolution ultrasound of the supraclavicular brachial plexus: can it improve therapeutic decisions in patients with plexus trauma? Eur Radiol 2007;17:1611-20 CrossRef Medline

22. Doi K, Otsuka $\mathrm{K}$, Okamoto $\mathrm{Y}$, et al. Cervical nerve root avulsion in brachial plexus injuries: magnetic resonance imaging classification and comparison with myelography and computerized tomography myelography. J Neurosurg 2002;96:277-84 Medline

23. Tharin BD, Kini JA, York GE, et al. Brachial plexopathy: a review of traumatic and nontraumatic causes. AJR Am J Roentgenol 2014;202: W67-75 CrossRef Medline

24. O'Brien DF, Park T, Noetzel MJ, et al. Management of birth brachial plexus palsy. Childs Nerv Syst 2006;22:103-12 CrossRef Medline

25. Greenwald AG, Schute PC, Shiveley JL. Brachial plexus birth palsy: a 10-year report on the incidence and prognosis. J Pediatr Orthop 1984;4:689-92 CrossRef Medline

26. Pondaag W, Malessy MJ, van Dijk JG, et al. Natural history of obstetric brachial plexus palsy: a systematic review. Dev Med Child Neurol 2004;46:138-44 CrossRef Medline 